\title{
Phase behaviour and orientational ordering in block copolymers doped with anisotropic nanoparticles.
}

\author{
M.A. Osipov ${ }^{1,2}$, M.V. Gorkunov ${ }^{3}$, A.V. Berezkin ${ }^{2}$, and Y.V. Kudryavtsev ${ }^{2,4}$ \\ 1 Department of Mathematics and Statistics, University of Strathclyde, Glasgow G1 1XH, Scotland, UK \\ 2 Topchiev Institute of Petrochemical Synthesis, Russian Academy of Sciences, 119991 Moscow, Russia \\ 3 Shubnikov Institute of Crystallography, Federal Scientific Research Centre "Crystallography and Photonics", \\ Russian Academy of Sciences, 119333 Moscow, Russia \\ 4 Frumkin Institute of Physical Chemistry and Electrochemistry, \\ Russian Academy of Sciences, 119071, Moscow, Russia
}

\begin{abstract}
A molecular field theory and coarse grained computer simulations with dissipative particle dynamics have been used to study the spontaneous orientational ordering of anisotropic nanoparticles in the lamellae and hexagonal phases of diblock copolymers and the effect of nanoparticles on the phase behaviour of these systems. Both a molecular theory and computer simulations indicate that strongly anisotropic nanoparticles are ordered orientationally mainly in the boundary region between the domains, and the nematic order parameter possesses opposite signs in adjacent domains. The orientational order is induced by the boundary and by the interaction between nanoparticles and the monomer units in different domains. In simulations, sufficiently long and strongly selective nanoparticles order also inside the domains. Nematic order parameter and local concentration profiles of nanoparticles have been calculated numerically using the model of a nanoparticle with two interaction centres and also determined using the results of computer simulations. A number of phase diagrams have been obtained which illustrate the effect of nanoparticle selectivity and molar fraction of the stability ranges of various phases. Different morphologies have been identified by analysing the static structure factor and a phase diagram has been constructed in coordinates nanoparticle concentration - copolymer composition. Orientational ordering of even a small fraction of nanoparticles may result in a significant increase of the dielectric anisotropy of a polymer nanocomposite which is important for various applications.
\end{abstract}

\section{INTRODUCTION}

Various soft matter nanocomposites based on anisotropic fluids and doped by metal, dielectric and semiconductor nanoparticles (NPs) are considered to be very promising materials which attract significant attention at present. To a large extent this interest is determined by a possibility to modify thermodynamic, optical and dielectric properties of such systems by adding a small fraction of NPs [1]. For example, switching voltages and times of nematic liquid crystal cells can be reduced by adding only a few volume percent of metal NPs (see, for example, Refs. [2-6]). Doping of nematics with strongly polar ferroelectric NPs also leads to an increase of their dielectric constant and enhances the electro-optic response [7-11]. In nanocomposites based on side-chain liquid crystal polymers, the semiconductor quantum dots may positionally order in self-assembled periodic structures $[12,13]$.

Anisotropic NPs acquire orientational order from various liquid crystal host phases and, in turn, affect the thermodynamic stability of these phases. Indeed, the nematic-isotropic (N-I) phase transition temperature is decreased when the nematic liquid crystal is doped with isotropic silver [14], gold [15] or aerosil [16, 17] NPs . At the same time, doping with strongly anisotropic NPs leads to an increase of the N-I transition temperature, which has been observed using nanotubes [18], magnetic nanorods (NRs) [19], and ferroelectric NPs [7, 8]. Recently, the effect of NPs on the N-I phase transition and on the dielectric properties of nematic nano-composites has been described in terms of molecular statistical theory [20-24].

Introduction of NPs can also improve mechanical, thermal, and dielectric properties of composite polymer nanomaterials based on block copolymers [25-31]. Microphase separation in block copolymers can stabilize spatially inhomogeneous NP distribution which paves the way for the design of materials to be used as smart membranes and in nanophotonics. The self-assembling of such nanostructures will be of advantage compared to the production by lithographic techniques. From the liquid crystal science viewpoint, interesting effects are related to the orientational ordering of small anisotropic NPs in various phases of block copolymers. Hexagonal and lamellae phases of block copolymers are macroscopically uniaxial, and this structural anisotropy can, in principle, induce some orientational order of sufficiently anisotropic NRs. On the other hand, the macroscopic anisotropy of diblock copolymers is expected to be rather weak because the bulk of each block is isotropic and the anisotropy is only determined by the block boundaries. As a result, NRs can order orientationally only in the interfacial regions between adjacent blocks, where they interact with the monomers of both blocks. The orientational ordering of NRs in diblock copolymers has been indeed observed experimentally. In particular, poly(ethylene glycol) functionalized gold NRs have been aligned parallel to the domain boundary in the lamellae phase [32], while the polystyrene functionalized NRs have been aligned per- 
pendicular to the cylindrical domain boundary in the hexagonal phase of the same block copolymer [33]. At the same time, the alkyl phosphonic acid capped semiconductor NPs have been ordered parallel to the cylindrical boundary in a different block copolymer [34, 35].

In general, the effect of NPs on the structure and properties of block copolymers can be rather complicated. On the one hand, anisotropic NPs may spontaneously order both spatially and orientationally in the anisotropic lamellae and hexagonal phases. On the other hand, they may also affect the thermodynamic stability of these phases and shift the corresponding transition points. The distribution of anisotropic NPs in block copolymers has recently been studied theoretically by the authors [36, 37] assuming fixed phase-separated structures of the lamellae and hexagonal phases. Similarly to the molecular theory of nematic liquid crystals in the external field, the orientational order of NPs in this case is induced by the anisotropic mean-field created by the interaction between the NPs and the monomers of the two different blocks. In the simplest model, a NP was assumed to be of spherical shape but its interaction with a monomer was a sum of the isotropic and anisotropic parts. The anisotropic part of the model potential was determined by the effective particle anisotropy due to, for example, the polarizability anisotropy or the quadrupole moment. Numerical calculations have been employed to show that anisotropic NPs are orientationally ordered in the interfacial regions [36] and that the NPs are aligned parallel and perpendicular to the domain wall on different sides of the boundary.

Coarse-grained simulations with dissipative particle dynamics (DPD) [37] allowed us to explicitly consider anisometric NPs (and NRs) and to study the effect of their concentration, length, stiffness, and selectivity of their interactions with the copolymer blocks on the orientational order of NPs and phase stability of the whole composite. In our recent theoretical paper [38], a dumbbell NP has been considered, which is composed of two equal spheres separated by a certain distance and interacting individually with the monomers. Such a model NP possesses an anisotropic shape and is rather similar to the model NR, composed of several rigidly connected spheres, which was employed in the computer simulations [37]. It has been shown that in the context of this model, the orientational order parameter of the NPs appears to be significantly higher than in the previous model [36] and the NPs are orientationally ordered in a broader interfacial region which is closer to the results of computer simulations.

In this paper, we use both analytical theory and computer simulations to focus on the effect of the NRs on the relative stability of the lamellae and hexagonal phases with respect to each other and to the isotropic phase. We combine the expansion of the free energy of the pure block copolymer in terms of the order parameters of the monomer distribution with a molecular-field theory which describes the interaction of the NRs with the monomers and their ordering in block copolymers. In parallel, we carry out extensive DPD simulations to construct partial phase diagrams of the composite in the coordinates NR molar fraction polymer composition at a fixed strong immiscibility of the copolymer blocks. Different morphologies are identified by analysing the static structure factor and visually from the system snapshots. Orientational and positional order of the NRs are evaluated in lamellar and hexagonal phases. The paper ends with a discussion section bridging the model theoretical and simulation results.

\section{MOLECULAR FIELD THEORY OF POLYMER NANOCOMPOSITES}

In the context of the existing molecular statistical theory of block copolymers, the polymer nanocomposites with anisotropic NPs can be described combining fieldtheoretical approaches, including the self-consistent field theory [39], with an explicit treatment of NPs using the density functional theory [40]. In principle, such an approach can be used to consider NPs of arbitrary shape and size, but the number of publications in this field is very limited [41] which is related to inherent problems associated with high computational cost and with the difficulties in combining numerical computer simulations with the solutions of complex partial differential equations with both translational and orientational degrees of freedom. On the other hand, at relatively weak segregation between different monomers the block copolymer matrix can be described qualitatively by using the Landau expansion of the free energy of the chains in terms of the translational order parameters of the monomers in the lamellae and hexagonal phases as first proposed by Leibler [42]. Although more sophisticated mean-field theories encompassing block copolymers with stronger segregation have been also developed [43, 44], we choose here to take the advantage of the simplicity and clarity of Leibler's approach and combine the free energy expansion in terms of the translational order parameter with the NP-monomer interaction accounted in the molecular field approximation.

Assuming that the molar fraction of NPs is sufficiently small, one can neglect the interaction of NPs and write the free energy density of a block copolymer doped with NPs with three terms:

$$
F=F_{p}+V_{N P}-T S_{N P},
$$

Here the free energy of pure host copolymer consists of the contribution from the entropy of non-interacting chains and of the repulsive interaction of monomers A and B:

$$
F_{p}=-k_{B} T \ln Z_{0}+k_{B} T \int \chi_{0}\left\langle\rho_{A}(\mathbf{r})\right\rangle\left\langle\rho_{B}(\mathbf{r})\right\rangle d \mathbf{r},
$$

where $Z_{0}$ is the chain partition function and $\chi_{0}>0$ is the effective monomer repulsion parameter. The energy 
of the NP interaction with the monomers $\mathrm{A}$ and $\mathrm{B}$ reads as:

$$
\begin{gathered}
V_{N P}=\int\left\langle\rho_{A}\left(\mathbf{r}_{1}\right)\right\rangle V_{A}\left(\mathbf{r}_{12}, \mathbf{a}_{2}\right) \rho_{N P}\left(\mathbf{r}_{2}, \mathbf{a}_{2}\right) d \mathbf{r}_{1} d \mathbf{r}_{2} d \mathbf{a}_{2} \\
+\int\left\langle\rho_{B}\left(\mathbf{r}_{1}\right)\right\rangle V_{B}\left(\mathbf{r}_{12}, \mathbf{a}_{2}\right) \rho_{N P}\left(\mathbf{r}_{2}, \mathbf{a}_{2}\right) d \mathbf{r}_{1} d \mathbf{r}_{2} d \mathbf{a}_{2}
\end{gathered}
$$

while the last term in Eq. (1) corresponds to the contribution of the orientational and translational entropy of anisotropic NPs:

$$
S_{N P}=k_{B} \int \rho_{N P}(\mathbf{r}, \mathbf{a}) \ln \left[\rho_{N P}(\mathbf{r}, \mathbf{a})\right] d \mathbf{r} d \mathbf{a} .
$$

We denote as $\rho_{A}(\mathbf{r})$ and $\rho_{B}(\mathbf{r})$ the density profiles of monomers of types $\mathrm{A}$ and $\mathrm{B}$ respectively, while $\rho_{N P}(\mathbf{r}, \mathbf{a})$ is the one-particle density describing the positional and orientational distribution of the NPs normalized by their number: $\quad N_{N P}=\int \rho_{N P}(\mathbf{r}, \mathbf{a}) d \mathbf{r} d \mathbf{a}$. The potentials $V_{A}\left(\mathbf{r}_{12}, \mathbf{a}_{2}\right)$ and $V_{B}\left(\mathbf{r}_{12}, \mathbf{a}_{2}\right)$ describe the interaction between an anisotropic NP and the monomers A and B, respectively, and they are assumed to depend on the distance $\mathbf{r}_{12}=\mathbf{r}_{1}-\mathbf{r}_{2}$ and the NP orientation specified by the unit vector $\mathbf{a}$ in the direction of the NP main axis.

\section{A. Free energy expansion of the pure host phase}

In the limit of zero polymer compressibility, $\rho_{A}(\mathbf{r})+$ $\rho_{B}(\mathbf{r})=\rho_{m}$, where $\rho_{m}$ is the average monomer number density. Then the order parameter of the microphase separated structure in a diblock copolymer can be introduced as:

$$
\psi(\mathbf{r})=\frac{1}{\rho_{m}}\left\langle\delta \rho_{A}\right\rangle=\frac{1}{\rho_{m}}\left\langle\rho_{A}\right\rangle-f=1-f-\frac{1}{\rho_{m}}\left\langle\rho_{B}\right\rangle,
$$

where $f$ is the relative composition ratio of the diblock copolymer chain. This order parameter can be sought in the form of the following general expression valid for different phases [42]:

$$
\psi(\mathbf{r})=n^{-1 / 2} \psi_{n} \sum_{j=1}^{n}\left[\exp \left(i \mathbf{Q}_{j} \cdot \mathbf{r}+\varphi_{n}\right)+c . c .\right]
$$

where $\psi_{n}$ is the amplitude and $\varphi_{n}$ is the phase and the density distribution is characterized by a set of $n$ wave vectors $\mathbf{Q}_{j}, j=1, \ldots, n$. In the lamellae phase, there is only one wave vector, i.e., $n=1$ and $\left|\mathbf{Q}_{1}\right|=q^{*}$. In the hexagonal phase, $n=3$ and all three wave vectors $\mathbf{Q}_{1,2,3}$ are expressed in terms of the same $q^{*}$ as $\mathbf{Q}_{1}=q^{*}(1,0,0), \mathbf{Q}_{2}=q^{*} / 2(-1, \sqrt{3}, 0)$ and $\mathbf{Q}_{3}=$ $q^{*} / 2(-1,-\sqrt{3}, 0)$

Now the free energy $F_{p}$ per chain of the pure copolymer host phase can be expanded in terms of the order parameter as originally proposed by Leibler [42]:

$$
\frac{F_{p}}{k_{B} T N_{c h}}=2 N\left(\chi_{s}-\chi\right) \psi_{n}^{2}-\alpha_{n} \psi_{n}^{3}+\beta_{n} \psi_{n}^{4}
$$

where $\chi=\rho_{m} \chi_{0}, N$ is the number of monomers in a polymer chain, so that the total number of polymer chains reads as $N_{c h}=N_{m} / N$, where $N_{m}=\rho_{m} V$ is the total monomer number. The coefficients of the expansion depend on the fraction $f$ of the monomers $\mathrm{A}$ and on the product $q R$ of the wave number $q$ and the gyration radius $R=s \sqrt{N / 6}$ of the polymer chain where $s$ is the monomer length (the Kuhn segment length). Explicit expressions for the coefficients $\chi_{s}, \alpha_{n}$ and $\beta_{n}$ are presented in [42]. In particular, the quadratic term in this expansion has the same mathematical form for both phases and the coefficient $\chi_{s}$ can be written in the form:

$$
\begin{aligned}
N \chi_{s}=\frac{1}{2} g_{1}(1, x) /\left\{g_{1}(f, x) g_{1}(1-f, x)-\right. \\
\left.\frac{1}{4}\left[g_{1}(1, x)-g_{1}(f, x)-g_{1}(1-f, x)\right]^{2}\right\},
\end{aligned}
$$

where $g_{1}(f, x)=2[f x+\exp (-f x)-1] / x^{2}$ is the Debye function which depends on the wavenumber $q$ of the phase separated structure via the nondimensional parameter $x=q^{2} R^{2}$. The equilibrium wavenumber $q^{*}$ at the transition into the disordered phase can be obtained by minimizing Eq. (8) with respect to $x$, and the higher order coefficients $\alpha_{1}, \alpha_{3}$ and $\beta_{1}, \beta_{3}$ are calculated using this equilibrium value $q^{*}$. One notes that it follows from the symmetry of the lamellae phase that $\alpha_{1}=0$.

The higher order coefficients in the free energy expansion have been explicitly expressed in [42]. In particular, in the lamellae phase

$$
\beta_{1}=N \Gamma_{4}(0,0) / 4
$$

and in the hexagonal phase

$$
\alpha_{3}=-\frac{2}{3 \sqrt{3}} N \Gamma_{3}
$$

and

$$
\beta_{3}=N\left[\Gamma_{4}(0,0)+4 \Gamma_{4}(0,1)\right] / 12 .
$$

The particular values of the parameters $\Gamma_{3}, \Gamma_{4}(0,0)$ and $\Gamma_{4}(0,1)$ used below are extracted from Ref. [42], where they have been evaluated for various copolymer configurations.

\section{B. NP contribution to the free energy of the composite}

The NP distribution function $\rho_{N P}(\mathbf{r}, \mathbf{a})$ can be obtained by minimizing the total free energy (1). Expressing the average monomer density in terms of the order 
parameter (5) and using Eq. (6), one obtains:

$$
\begin{gathered}
\rho_{N P}\left(\mathbf{r}_{2}, \mathbf{a}_{2}\right)=\frac{1}{Z} \exp \left\{-\frac{\rho_{m}}{k_{B} T} \int \psi\left(\mathbf{r}_{1}\right) \times\right. \\
\left.\left[V_{A}\left(\mathbf{r}_{12}, \mathbf{a}_{2}\right)-V_{B}\left(\mathbf{r}_{12}, \mathbf{a}_{2}\right)\right] d \mathbf{r}_{1}\right\}= \\
=\frac{1}{Z} \exp \left\{-\frac{\rho_{m}}{k_{B} T} n^{-1 / 2} \psi_{n} \times\right. \\
\left.\sum_{j=1}^{n}\left[\exp \left(i \mathbf{Q}_{j} \cdot \mathbf{r}_{2}\right) \delta V\left(\mathbf{Q}_{j}, \mathbf{a}_{2}\right)+\text { c.c. }\right]\right\}
\end{gathered}
$$

where $\delta V\left(\mathbf{Q}_{i}, \mathbf{a}_{2}\right)=\int\left[V_{A}\left(\mathbf{r}_{12}, \mathbf{a}_{2}\right)-V_{B}\left(\mathbf{r}_{12}, \mathbf{a}_{2}\right)\right] \exp \left(i \mathbf{Q}_{i}\right.$ $\left.\mathbf{r}_{12}\right) d \mathbf{r}_{12}$

Substituting this expression into the free energy (1) one obtains the following contribution of NPs to the total free energy:

$$
\begin{aligned}
\frac{F_{N P}}{V k_{B} T}= & -\rho_{N P} \ln Z= \\
= & -\rho_{N P} \ln \int \exp \left\{-\frac{\rho_{m}}{k_{B} T} n^{-1 / 2} \psi_{n} \times\right. \\
& \left.\sum_{i=1}^{n}\left[\exp \left(i \mathbf{Q}_{i} \cdot \mathbf{r}\right) \delta V\left(\mathbf{Q}_{i}, \mathbf{a}\right)+c . c .\right]\right\} d \mathbf{r} d \mathbf{a}
\end{aligned}
$$

where $\rho_{N P}$ is the average number density of NPs, and $V$ is the system volume.

The free energy contribution determined by NPs can now be expanded in powers of $\psi_{n}$ using the equations for the order parameter $\psi(\mathbf{r})$ for the lamellae and hexagonal phases:

$$
\frac{F_{N P}}{V k_{B} T}=-\rho_{N P} \sum_{k} \frac{\rho_{m}^{k}}{k !\left(k_{B} T\right)^{k}} n^{-k / 2} G_{k} \psi_{n}^{k},
$$

where

$$
\begin{array}{r}
G_{1}=I_{1}, \quad G_{2}=I_{2}-I_{1}^{2}, \quad G_{3}=I_{3}-3 I_{1} I_{2}+2 I_{1}^{3}, \\
G_{4}=I_{4}-4 I_{1} I_{3}-3 I_{2}^{2}+12 I_{1}^{2} I_{2}-6 I_{1}^{4},
\end{array}
$$

and where

$$
\begin{aligned}
I_{k} & =\frac{1}{4 \pi V} \times \\
& \int\left[\sum_{j=1}^{n}\left[\exp \left(i \mathbf{Q}_{j} \cdot \mathbf{r}\right) \delta V\left(\mathbf{Q}_{j}, \mathbf{a}\right)+\text { c.c. }\right]\right]^{k} d \mathbf{r} d \mathbf{a} .
\end{aligned}
$$

One notes that in this expression only the exponential functions $\exp \left(i \mathbf{Q}_{j} \cdot \mathbf{r}\right)$ are to be integrated over $\mathbf{r}$ which makes the integration rather straightforward.

For example, in the lamellae phase, $n=1$ and there is a single nonzero wave number $\left|\mathbf{Q}_{1}\right|=q^{*}$. In this case, the quantities $I_{i}$ and $G_{i}$ are given by the following expressions:

$$
\begin{array}{r}
I_{1,3}^{(l)}=0, I_{2}^{(l)}=\frac{1}{2 \pi} \int \delta V\left(\mathbf{Q}_{1}, \mathbf{a}\right) \delta V\left(-\mathbf{Q}_{1}, \mathbf{a}\right) d \mathbf{a} \\
I_{4}^{(l)}=\frac{3}{2 \pi} \int\left[\delta V\left(\mathbf{Q}_{1}, \mathbf{a}\right) \delta V\left(-\mathbf{Q}_{1}, \mathbf{a}\right)\right]^{2} d \mathbf{a}
\end{array}
$$

and, accordingly, $G_{1,3}^{(l)}=0, G_{2}^{(l)}=I_{2}^{(l)}, G_{4}^{(l)}=I_{4}^{(l)}-$ $3 I_{2}^{(l)^{2}}$.

In the hexagonal phase, $n=3$ and there are three wave vectors which have the same absolute value $\left|\mathbf{Q}_{1,2,3}\right|=q^{*}$ and $\mathbf{Q}_{1}+\mathbf{Q}_{2}+\mathbf{Q}_{3}=\mathbf{0}$. In this case, one also obtains $I_{1}^{(h)}=0$ and the even-order integrals are very similar to those obtained in the case of the lamellae phase. At the same time, the third-order integral $I_{3}^{(h)}$ is nonzero as the product of the three exponential functions does not vanish upon integration over $\mathbf{r}$ if it involves all three different wave vectors. As a result, one obtains:

$$
\begin{gathered}
I_{2}^{(h)}=\frac{1}{2 \pi} \sum_{m=1}^{3} \int \delta V\left(\mathbf{Q}_{m}, \mathbf{a}\right) \delta V\left(-\mathbf{Q}_{m}, \mathbf{a}\right) d \mathbf{a} \\
I_{3}^{(h)}=\frac{3}{2 \pi} \int\left[\delta V\left(\mathbf{Q}_{1}, \mathbf{a}\right) \delta V\left(\mathbf{Q}_{2}, \mathbf{a}\right) \delta V\left(\mathbf{Q}_{3}, \mathbf{a}\right)+\text { c.c. }\right] d \mathbf{a} \\
I_{4}^{(h)}=\frac{3}{2 \pi} \sum_{m=1}^{3} \int\left[\delta V\left(\mathbf{Q}_{m}, \mathbf{a}\right) \delta V\left(-\mathbf{Q}_{m}, \mathbf{a}\right)\right]^{2} d \mathbf{a}
\end{gathered}
$$

where the expressions for $I_{2}^{(h)}$ and $I_{4}^{(h)}$ are composed of three equal contributions, and $I_{2}^{(h)}=3 I_{2}^{(l)}, I_{4}^{(h)}=3 I_{4}^{(l)}$. The free energy expansion coefficients in the hexagonal phase are $G_{1}^{(h)}=0, G_{2}^{(h)}=3 I_{2}^{(l)}, G_{3}^{(h)}=I_{3}^{(h)}, G_{4}^{(h)}=$ $3 I_{4}^{(l)}-27 I_{2}^{(l)^{2}}$.

Therefore, in both phases, the free energy expansion coefficients are expressed in terms of the three integrals of the Fourier transforms of the potential: $I_{2}^{(l)}, I_{4}^{(l)}$, and $I_{3}^{(h)}$. More detailed expressions can be obtained using a particular model of the interaction potential. In this paper we use the dumbbell model of the nanoparticle which is composed of the two spheres with their centres separated by a distance $l$. The spheres interact isotropically with monomers via the exponential potential $V_{\alpha}(r)=J_{\alpha} \exp \left(-r^{2} / r_{0}^{2}\right)$ where $r$ is the distance between the centre of the sphere and the monomer of the type $\alpha=A, B$. In this case, the energy of interaction of a NP composed of two spheres with a monomer is expressed as

$$
V_{\alpha}\left(\mathbf{r}_{12}, \mathbf{a}\right)=J_{\alpha} \exp \left(-r_{+}^{2} / r_{0}^{2}\right)+J_{\alpha} \exp \left(-r_{-}^{2} / r_{0}^{2}\right),
$$

where $\mathbf{r}_{+}=\mathbf{r}_{12}+l \mathbf{a} / 2, \mathbf{r}_{-}=\mathbf{r}_{12}-l \mathbf{a} / 2$. Here the unit vector $\mathbf{a}$ is parallel to the NP axis.

The Fourier transform of this potential can be evaluated in an explicit form:

$$
\begin{gathered}
V_{\alpha}^{+}(\mathbf{Q}, \mathbf{a})=\int J_{\alpha} \exp \left(-r_{+}^{2} / r_{0}^{2}\right) \exp \left(i \mathbf{Q} \cdot \mathbf{r}_{12}\right) d \mathbf{r}_{12}= \\
=\exp (-i \mathbf{Q} \cdot \mathbf{a} l / 2) \int J_{\alpha} \exp \left(-r_{+}^{2} / r_{0}^{2}\right) \exp \left(i \mathbf{Q} \cdot \mathbf{r}_{+}\right) d \mathbf{r}_{+}= \\
=\pi^{3 / 2} r_{0}^{3} J_{\alpha} \exp (-i \mathbf{Q} \cdot \mathbf{a} l / 2) \exp \left(-Q^{2} r_{0}^{2} / 4\right), \quad(23)
\end{gathered}
$$


and hence

$$
\begin{aligned}
\delta V(\mathbf{Q}, \mathbf{a})=2 \pi^{3 / 2} & r_{0}^{3}\left(J_{A}-J_{B}\right) \times \\
& \cos (\mathbf{Q} \cdot \mathbf{a} l / 2) \exp \left(-Q^{2} r_{0}^{2} / 4\right) .
\end{aligned}
$$

Then the quantities relevant for the free energy coefficients in the lamellae and hexagonal phases take the form:

$$
I_{2}^{(l)}=4 \pi^{3} r_{0}^{6}\left(J_{A}-J_{B}\right)^{2} \exp \left(-q^{* 2} r_{0}^{2} / 2\right)\left(1+\frac{\sin q^{*} l}{q^{*} l}\right),
$$

$$
\begin{gathered}
I_{4}^{(l)}=6 \pi^{6} r_{0}^{12}\left(J_{A}-J_{B}\right)^{4} \exp \left(-q^{* 2} r_{0}^{2}\right) \times \\
\left(6+\frac{8 \sin q^{*} l+\sin 2 q^{*} l}{q^{*} l}\right), \\
I_{3}^{(h)}=96 \pi^{9 / 2} r_{0}^{9}\left(J_{A}-J_{B}\right)^{3} \exp \left(-3 q^{* 2} r_{0}^{2} / 4\right) .
\end{gathered}
$$

Note that the latter integral is independent of the NP anisotropy.

\section{PHASE TRANSITIONS OF THE COMPOSITE}

The total free energy of the copolymer-NP composite is given by the sum of the polymer chains free energy (7) and the NP contribution (14), $F=F_{p}+F_{N P}$. The free energy per chain for the lamellae phase can be written in the form of the following expansion:

$$
\frac{F^{(l)}}{k_{B} T N_{c h}}=a \psi_{1}^{2}+c^{(l)} \psi_{1}^{4} .
$$

The free energy expansion in the hexagonal phase can be expressed as:

$$
\frac{F^{(h)}}{k_{B} T N_{c h}}=a \psi_{3}^{2}-b \psi_{3}^{3}+c^{(h)} \psi_{3}^{4},
$$

where the quadratic term

$$
a=2 N\left(\chi_{s}-\chi\right)-\frac{N \rho_{N P} \rho_{m}}{2\left(k_{B} T\right)^{2}} I_{2}^{(l)}
$$

is the same for both phases. The third order coefficient is nonzero only in the hexagonal phase:

$$
b=\alpha_{3}-\frac{N \rho_{N P} \rho_{m}^{2}}{18 \sqrt{3}\left(k_{B} T\right)^{3}} I_{3}^{(h)}
$$

The fourth order coefficients are different in different phases:

$$
\begin{aligned}
& c^{(l)}=\beta_{1}-\frac{N \rho_{N P} \rho_{m}^{3}}{24\left(k_{B} T\right)^{4}}\left(I_{4}^{(l)}-3 I_{2}^{(l)^{2}}\right), \\
& c^{(h)}=\beta_{3}-\frac{N \rho_{N P} \rho_{m}^{3}}{72\left(k_{B} T\right)^{4}}\left(I_{4}^{(l)}-9 I_{2}^{(l)^{2}}\right),
\end{aligned}
$$

and should stay positive to ensure the system stability.

Equations (28) and (29) can now be used to evaluate the free energies of different phases. At the first stage, the coefficient $a$ is minimized to obtain the equilibrium microphase separation wavenumber $q^{*}$. This wavenumber is then substituted into other coefficients and finally the free energies are to be minimized with respect to the corresponding order parameters.

The lamellae phase is thermodynamically profitable compared to the disordered phase as long as

$$
a<0 \text {. }
$$

Indeed, then the equilibrium order parameter $\tilde{\psi}_{1}=$ $\sqrt{-a / 2 c^{(l)}}$ minimizes the corresponding free energy and the latter being expressed as

$$
\frac{F_{e q}^{(l)}}{k_{B} T}=-\frac{a^{2}}{4 c^{(l)}}
$$

is always lower than the free energy of the disordered phase $F=0$.

The hexagonal phase corresponds to a local free energy minimum with nonzero $\psi_{3}$ when $a<9 / 8 b^{2} / 4 c^{(h)}$. However, it is favourable compared to the disordered state only when

$$
a<\frac{b^{2}}{4 c^{(h)}} .
$$

The equilibrium order parameter of the hexagonal phase corresponding to the global minimum of the free energy (29) and can be obtained by direct minimization:

$$
\tilde{\psi}_{3}=\frac{3 b}{8 c^{(h)}}\left(1+\sqrt{1-\frac{32 a c^{(h)}}{9 b^{2}}}\right)
$$

The equilibrium free energy of the hexagonal phase can be expressed as

$$
\frac{F_{e q}^{(h)}}{k_{B} T}=-\frac{1}{3} \tilde{\psi}_{3}^{2}\left(c^{(h)} \tilde{\psi}_{3}^{2}-a\right) .
$$

Accordingly, the hexagonal phase has the lower free energy compared to the lamellae phase when

$$
\frac{3 a^{2}}{4 c^{(l)}}<\tilde{\psi}_{3}^{2}\left(c^{(h)} \tilde{\psi}_{3}^{2}-a\right) .
$$

Thus the phase boundaries are determined by the lines defined by Eqs. (34), (36), and (39) which generally are to be resolved numerically. At the same time, the above relations can be used to derive some qualitative conclusions. In particular, one can readily see from Eq. (30) that the NPs destabilize the isotropic phase due to the last term, which is proportional to the NP number density, and is negative if $I_{2}>0$. The physical meaning of this contribution is related to the effective interaction between two monomers via a NP which increases the effective monomer interaction constant $\chi^{*}=\chi+\frac{N \rho_{N P} \rho_{m}}{2\left(k_{B} T\right)^{2}} I_{2}^{(l)}$. 

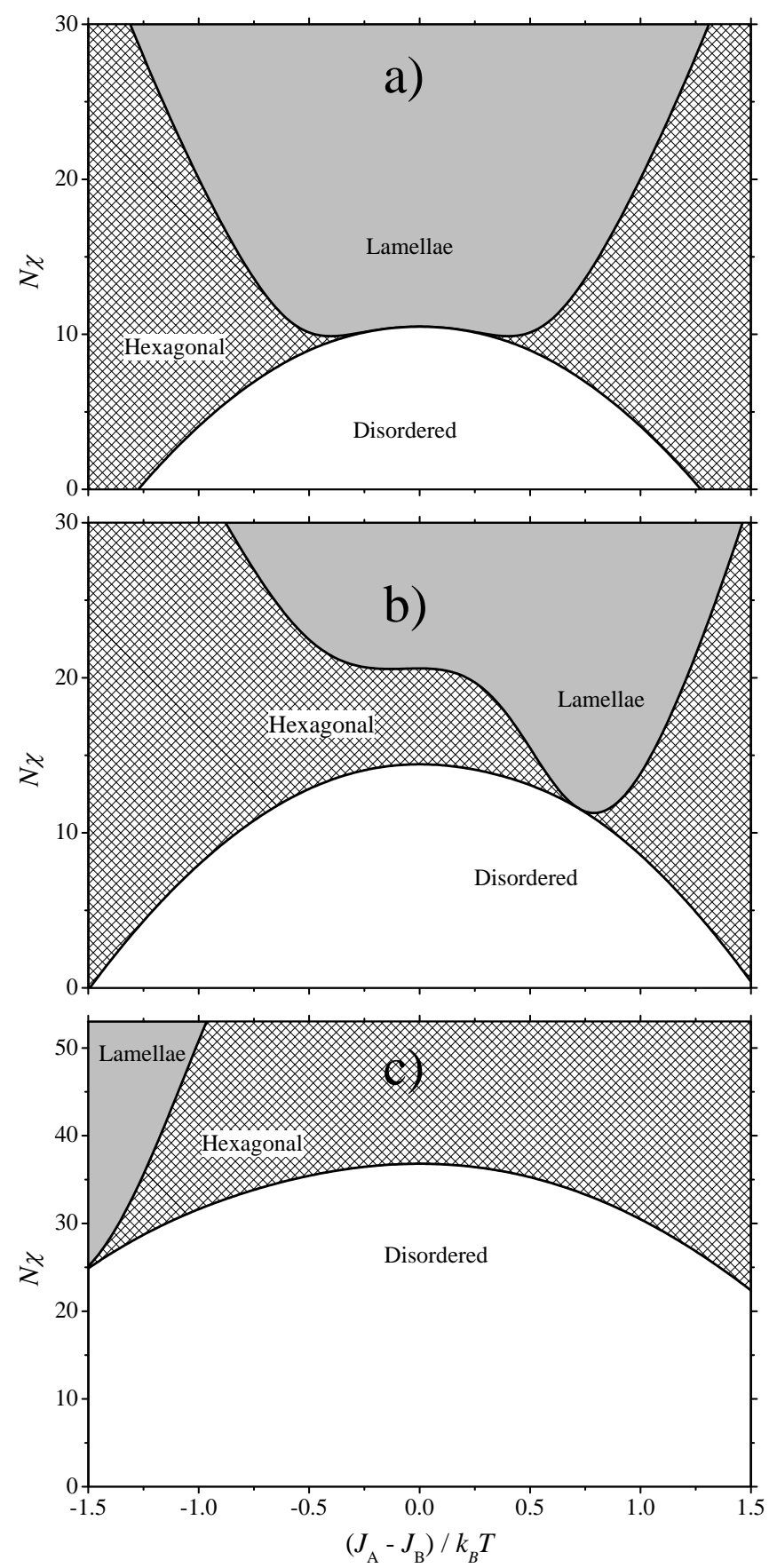

FIG. 1. Phase diagrams in terms of the monomer-monomer discrimination coupling constant $N \chi$ and the NP-monomer discrimination interaction constant $\left(J_{A}-J_{B}\right) / k_{B} T$ for different composition ratios: $f=0.5(\mathrm{a}), f=0.3(\mathrm{~b})$, and $f=0.15$ (c). The other model parameters are: $\rho_{N P} / \rho_{m}=10^{-3}$, $N=100, r_{0}=0.1 R, l=0.3 R, s=0.1 R, \rho_{m} r_{0}^{3}=1$.

\section{A. Model phase behaviour}

There are several length scales in the present model which have different orders of magnitude. Firstly, there
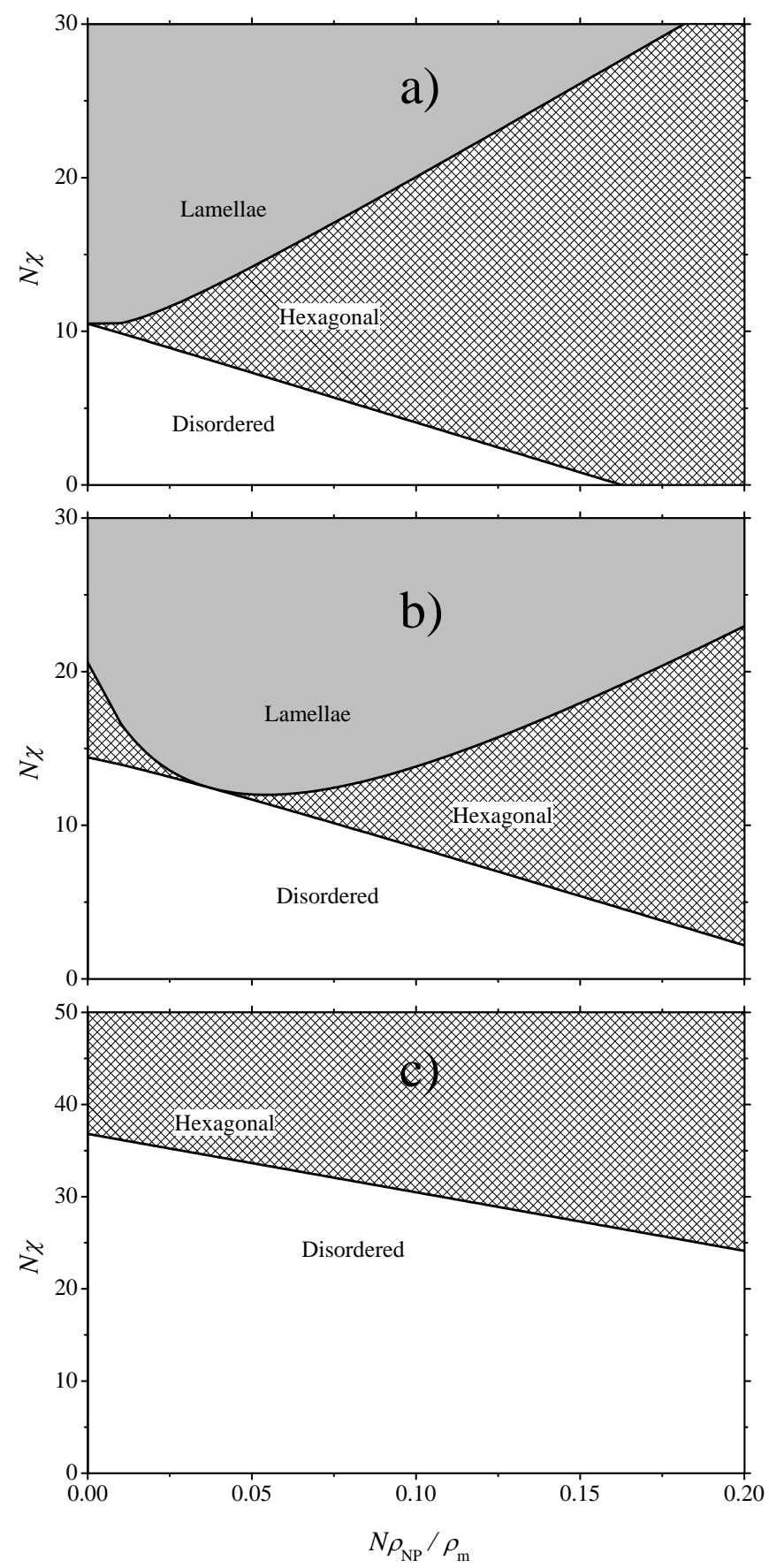

FIG. 2. Phase diagrams in terms of the monomer-monomer discrimination coupling constant $N \chi$ and the reduced NP number density $\rho_{N P} / \rho_{m}$ for different composition ratios: $f=$ $0.5(\mathrm{a}), f=0.3(\mathrm{~b})$, and $f=0.15$ (c). The other model parameters are: $N=100, r_{0}=0.1 R, l=0.3 R, s=0.1 R$, $\rho_{m} r_{0}^{3}=1, J_{A}-J_{B}=k_{B} T$.

are two polymer scales: the polymer segment length $s$ and the chain gyration radius $R=s \sqrt{N / 6}$, which is considerably larger than $s$. The microphase separated phases are characterized by the wavenumber $q^{*} \approx \sqrt{3.6} / R$ and 
hence the periodicity in the lamellae phase, for example, is about $\sim 3 R$, which means that $R$ is a good estimate for the microdomain size. The dumbbell model of a NP, considered above, is characterized by the length $l+2 r_{0}$ and thickness $2 r_{0}$. We assume that the NP length is smaller than the domain size, i.e. $l+2 r_{0}<R$, while the NP thickness is of the order of the monomer size, $r_{0} \sim s<<R$. Estimating the monomer density as $\rho_{m} \sim s^{-3}$, we can set the product $\rho_{m} r_{0}^{3} \sim 1$ which enters the coefficients $(30)-(33)$.

Phase diagrams of the nanocomposite in the coordinates $\left(N \chi,\left(J_{A}-J_{B}\right) / k T\right)$ and $\left(N \chi, N \rho_{N P} / \rho_{m}\right)$ are presented in Figs. 1 and 2 for different values of $f$ with the corresponding values of $N \Gamma_{3}$ and $N \Gamma_{4}$ taken from Leibler's plots [42]. One can readily see from Fig. 1 that NPs generally increase the stability of the hexagonal phase and decrease that of the disordered phase. In particular, in a symmetric block copolymer with $f=0.5$ (see Fig. 1a) the stability range of the hexagonal phase is rapidly increasing with the increasing discrimination interaction constant $\left(J_{A}-J_{B}\right)$ between the monomers and NPs regardless of its sign. Indeed, in this case, the profiles of the number densities of the monomers A and B are identical, and it plays no role which of them interact stronger with the NPs. In contrast, in a very asymmetric composite with $f=0.15$ (see Fig. 1c) ) the stability range of the hexagonal phase is increasing for positive $\left(J_{A}-J_{B}\right)$ and decreasing for negative $\left(J_{A}-J_{B}\right)$. This is related to the fact that the monomers $\mathrm{A}$ dominate in such composite and hence the stability range is increased only if the energy of interaction between the monomers $A$ and the NPs is lower than that between the NPs and monomers B, i.e., if $\left(J_{A}-J_{B}\right)>0$. In the intermediate case with $f=0.3$ presented in Fig. 1b), the phase diagram is more complicated as there are several competing contributions to the coefficients of the free energy expansion of the pure block copolymer which strongly depend on $f$.

Numerical results presented in Fig. 2 also indicate that generally the stability range of the hexagonal phase is increasing with the increasing concentration of NPs. A more complicated behaviour is again presented in Fig. $2 \mathrm{~b}$ ) for $f=0.3$. In this case, the stability range is decreasing at small number density of NPs and increasing at larger values of $\rho_{N P}$.

A typical dependence of the translational order parameters on the parameter $N \chi$ in the lamellae phase $\left(\Psi_{1}\right)$ and in the hexagonal phase $\left(\Psi_{3}\right)$ is presented in Fig. 3. It is interesting to note, that the order parameter $\Psi_{3}$ is positive for $\left(J_{A}-J_{B}\right)<0$ and negative for $\left(J_{A}-J_{B}\right)>0$, which is determined by the sign reversal of the coefficient $b$ in Eq. (37). The NP-related contribution to $b$ is a cubic function of $\left(J_{A}-J_{B}\right)$ according to Eqs. (31) and (27).
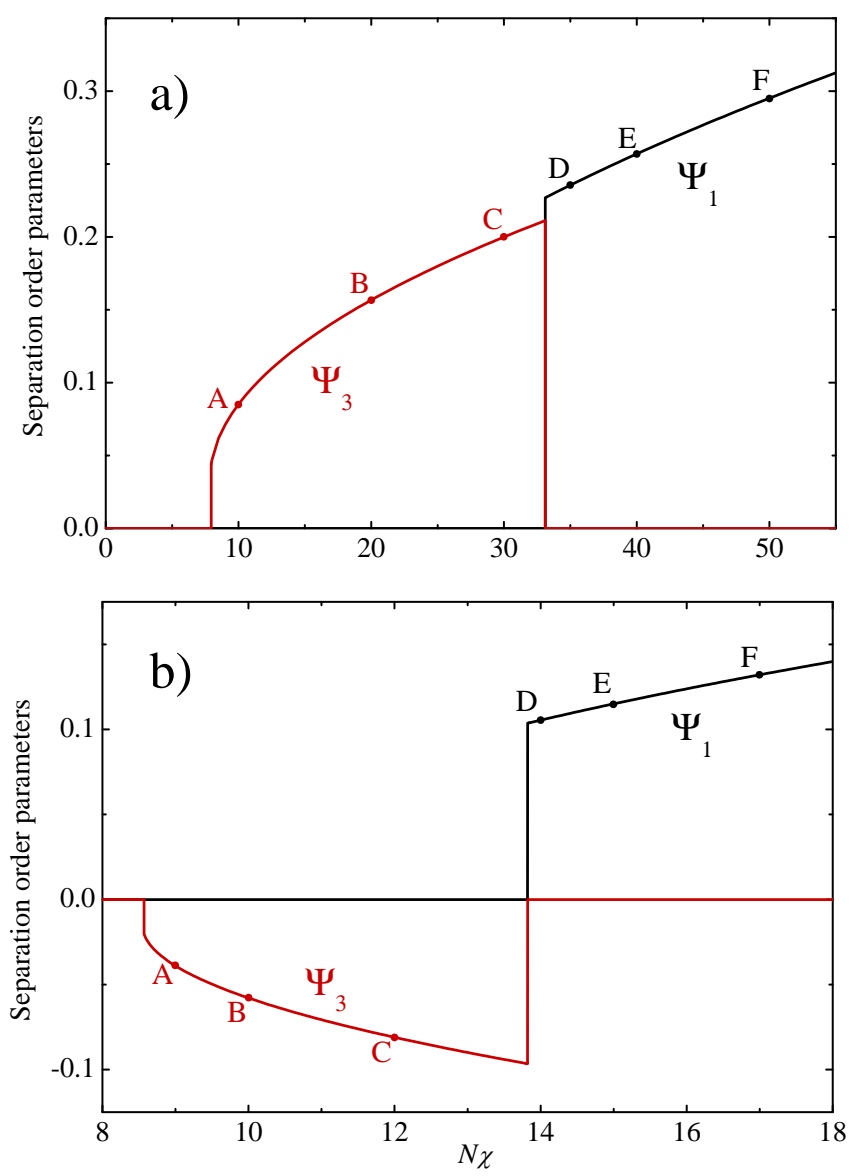

FIG. 3. Translational order parameters of the nano-composite for negative $\left(J_{A}-J_{B}\right)=-k_{B} T(\mathrm{a})$ and positive $\left(J_{A}-J_{B}\right)=$ $k_{B} T$ (b) values of the discrimination interaction constant and for $\rho_{N P}=10^{-3} \rho_{m}$ NPs. The other model parameters are: $N=100, r_{0}=0.1 R, l=0.3 R, s=0.1 R, \rho_{m}=s^{-3}$.

\section{B. Orientational and translational distribution of NPs}

Spatial distribution of anisotropic NPs and their orientational order is described by the one-particle distribution function (12) where the discrimination interaction potential $\delta V(\mathbf{Q}, \mathbf{a})$ is given by Eq. (24). In the lamellae phase, the density distribution of NPs is given by the following distribution function normalized over the period $\Lambda_{1}=2 \pi / q^{*}$ of the lamellae structure:

$$
f(x)=\frac{\int d \mathbf{a} \rho_{N P}(x, \mathbf{a})}{\int_{0}^{\Lambda_{1}} d x \int d \mathbf{a} \rho_{N P}(x, \mathbf{a})},
$$

where the $x$-axis is parallel to the wave vector of the periodic structure, $\mathbf{Q}_{1}=q^{*}(1,0,0)$.

The local orientational order parameter of NPs in the lamellae phase is expressed as:

$$
S(x)=\frac{\int d \mathbf{a} P_{2}(\mathbf{a} \cdot \mathbf{x}) \rho_{N P}(x, \mathbf{a})}{\int d \mathbf{a} \rho_{N P}(x, \mathbf{a})} .
$$



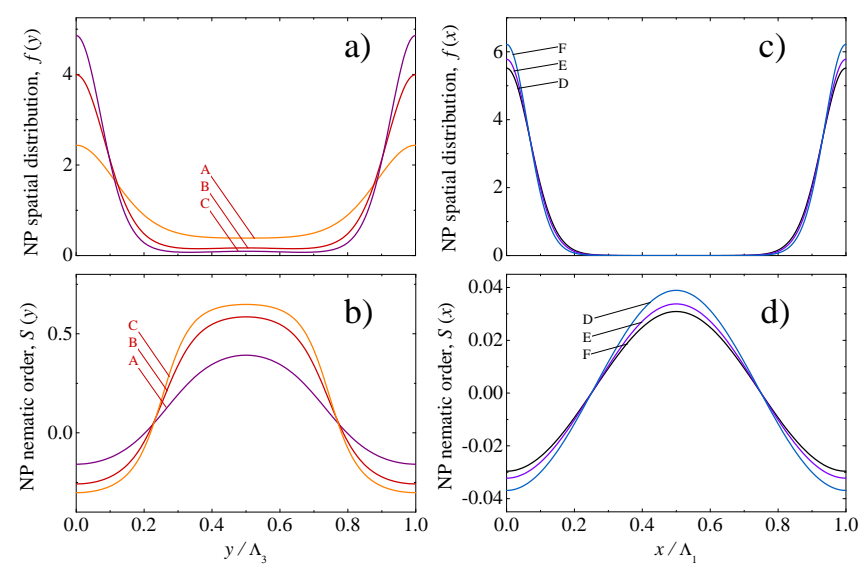

FIG. 4. Density $(a, c)$ and orientational order parameter profiles $(b, d)$ of the anisotropic NPs in the hexagonal $(a, b)$ and the lamellae $(c, d)$ phases evaluated at the points marked in Figure 3a) for negative $\left(J_{A}-J_{B}\right)=-k_{B} T$ and for $N=100$, $r_{0}=0.1 R, l=0.3 R, s=0.1 R, \rho_{m}=s^{-3}$.

The spatial and orientational distribution of NPs is illustrated in Figs. 4b) and 5b), where the density and the order parameter profiles along the $x$-axis are presented.

The hexagonal phase is characterized by a triangular 2D density distribution of both monomers and NPs specified by the wave vectors $\mathbf{Q}_{1}=q^{*}(1,0,0)$, $\mathbf{Q}_{2,3}=q^{*}(-1, \pm \sqrt{3}, 0) / 2$ and the spatial periodicity $\Lambda_{3}=4 \pi / \sqrt{3} q^{*}$ where the $z$-axis is parallel to the symmetry axes of the whole structure. The distribution of NPs in the hexagonal phase is illustrated in Figs. 4a) and 5a) where the density and the order parameter profiles along the axis $y($ at $x=0)$ are presented. These profiles are determined by the following equations:

$$
\begin{gathered}
f(y)=\frac{\int d \mathbf{a} \rho_{N P}(x=0, y, \mathbf{a})}{\int_{0}^{\Lambda_{3}} d y \int d \mathbf{a} \rho_{N P}(x=0, y, \mathbf{a})}, \\
S(y)=\frac{\int d \mathbf{a} P_{2}(\mathbf{a} \cdot \mathbf{y}) \rho_{N P}(x=0, y, \mathbf{a})}{\int d \mathbf{a} \rho_{N P}(x=0, y, \mathbf{a})} .
\end{gathered}
$$

One can readily see in Figs. 4 and 5 that the orientational order parameter of NPs possesses opposite signs in different domains of the copolymer in both the lamellae and the hexagonal phases. Similar behaviour has also been predicted in our previous paper [36] using a simple model where the distribution of monomers has been fixed. One notes that for a given set of parameters the NPs are predominantly located in one of the domains where they stronger interact with the corresponding monomers. Thus the majority of NPs in the composite are aligned along the same direction which should result in a sufficiently large dielectric and optical anisotropy of the nanocomposite.
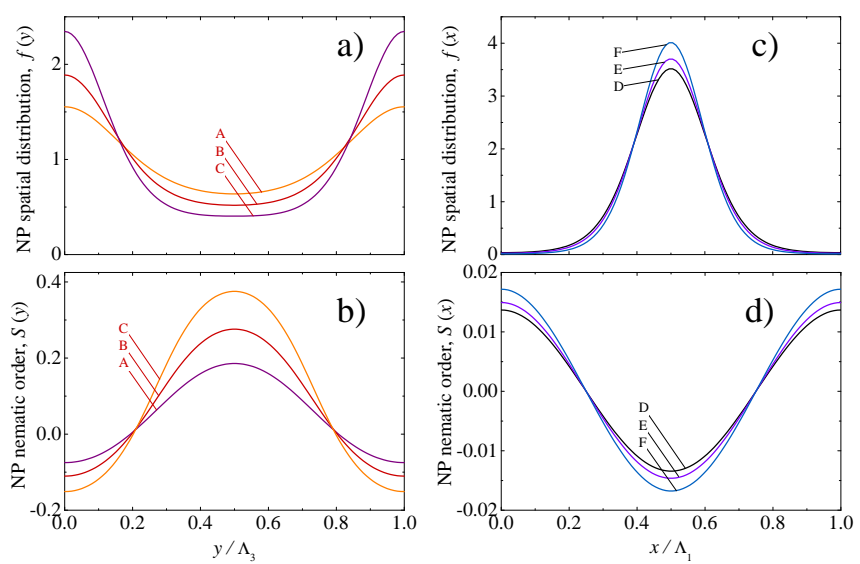

FIG. 5. Density $(a, c)$ and orientational order parameter profiles $(b, d)$ of the anisotropic NPs in the hexagonal $(a, b)$ and the lamellae $(c, d)$ phases evaluated at the points marked in Figure $3 \mathrm{~b})$ for positive $\left(J_{A}-J_{B}\right)=k_{B} T$ and for $N=100$, $r_{0}=0.1 R, l=0.3 R, s=0.1 R, \rho_{m}=s^{-3}$.

\section{COMPUTER SIMULATIONS}

Since its inception, the DPD method has become very popular in soft matter simulations, in particular, as a powerful tool to search for the microstructures in various block copolymer systems (see Ref. [45] and references therein). When it comes to composites, computational advantages of DPD only increase [50]. In the recent paper [37] we have used DPD simulations to study spatial and orientational ordering of NRs in the lamellar phase formed by a diblock copolymer $\mathrm{AB}$ of symmetric composition. Here we use the same method to encompass host matrices formed by asymmetric copolymers. It will enable us to describe the behaviour of anisotropic NPs in other phases, such as hexagonal and spherical ones. Aside from studying the arrangement of NRs in copolymer domains, we will demonstrate that in some cases their addition can qualitatively change the copolymer morphology itself.

\section{A. The model and the simulation method}

We consider the same coarse-grained model of a melted diblock copolymer doped with NRs as in Ref. [37]. A fully periodic simulation box of the size $l_{x} \times l_{y} \times l_{z}=24 \times 24 \times$ $24 r_{c}^{3}$ is filled with a total of 41472 DPD particles of three kinds, $A, B$, and $R$ to attain the average particle density $\rho_{0}=3 r_{c}^{-3}$ where $r_{c}$ is the unit length. The particles are soft and interact via conservative, dissipative, and random forces which are pairwise additive. The net force $\mathbf{F}_{i}=\sum_{j}\left(\mathbf{F}_{i j}^{C}+\mathbf{F}_{i j}^{D}+\mathbf{F}_{i j}^{R}\right)$ acting on a given particle $i$ is calculated as a sum over the forces from all other particles within the cut-off radius $r_{c}$. In what follows, we use dimensionless variables by setting $r_{c}$, the particle mass 
$m$, and $k_{B} T$ as the unit distance, mass, and thermal energy, respectively. This also defines the unit time as $\tau_{0}=r_{c}\left(m / k_{B} T\right)^{1 / 2}$.

Copolymer is modeled as a chain of 20 particles grouped into two blocks, $A$ and $B$, and connected via Hookean springs. Thus $A_{10} B_{10}$ is a symmetric copolymer with equal blocks $N_{A}=N_{B}=10$, whereas $A_{5} B_{15}$ has the shorter $A$-block with $N_{A}=5$ and the longer $B$-block with $N_{B}=15$. All NRs are composed of $N_{R}=5$ particles connected by rigid bonds of the constant length $b_{R}=0.7 r_{c}$. As found in Ref. [37], shorter NRs do not exhibit substantial ordering, whereas longer ones (even at $N_{R}=7$ ) demonstrate a tendency to macrophase separation. The equations of particle motion, $d \mathbf{r}_{i} / d t=\mathbf{v}_{i}, d \mathbf{v}_{i} / d t=\mathbf{F}_{i}$ are solved numerically using a free source code LAMMPS [51] that implements the modified velocity-Verlet algorithm (DPD-VV integration scheme) [52] with a time step $\delta t=0.02$.

For the copolymer, the conservative force represents the excluded volume interactions and elastic interactions of particles $i$ and $j$ in the dimensionless form $\mathbf{F}_{i j}^{C}=$ $a_{i j}\left(1-r_{i j}\right) \hat{\mathbf{r}}_{i j}-k_{s} \mathbf{r}_{i j}$ where $\mathbf{r}_{i j}=\mathbf{r}_{i} \mathbf{r}_{j}, r_{i j}=\left|\mathbf{r}_{i j}\right|, \hat{\mathbf{r}}_{i j}=$ $\mathbf{r}_{i j} / r_{i j}, a_{i j}$ is a maximum repulsion between the particles located at $\mathbf{r}_{i}=\mathbf{r}_{j}$ and $k_{s}$ is a spring constant which is taken to be $k_{s}=4$ for particles linked in a polymer chain and which is equal to zero for non-bonded particles. The dissipative and random forces, $\mathbf{F}_{i j}^{D}=$ $-\gamma \omega\left(r_{i j}\right)^{2}\left(\hat{\mathbf{r}}_{i j} \cdot \mathbf{v}_{i j}\right) \hat{\mathbf{r}}_{i j}$ and $\mathbf{F}_{i j}^{R}=\sigma \omega\left(r_{i j}\right) \xi(\delta t)^{-1 / 2} \hat{\mathbf{r}}_{i j}$, respectively, constitute the Groot-Warren thermostat [53], where $\gamma$ is a friction coefficient related to a thermal noise amplitude $\sigma$ via the fluctuation-dissipation theorem, $\gamma^{2}=2 \sigma, \omega(r)$ is a weight function, $\xi$ is a normally distributed random variable with zero mean and unit variance, which is uncorrelated for different particle pairs, $\delta t$ is the time step of an integration scheme, and $\mathbf{r}_{i j}=\mathbf{r}_{i}-\mathbf{r}_{j}$ is the relative velocity of particles $i$ and $j$. Following Ref. [53], we choose $\sigma=3$ and $\omega(r)=1-r$.

NRs are simulated as rigid bodies in the $N V E$ ensemble using an algorithm of Miller et al. [54]. Their correct temperature is maintained via the interactions with the surrounding thermostat composed of the polymeric DPD liquid. For comparison, we perform some simulations by replacing NRs with flexible chains consisting of $N_{R}=5$ particles of the $R$-type.

Miscibilities of the system components, i.e., the monomer $A$ units, monomer $B$ units and the $R$ NRs is determined by the differences $\delta a_{\alpha \beta}=a_{\alpha \beta}-a_{\alpha \alpha}$ $(\alpha, \beta=A, B, R)$ of the coefficients which describe the excluded volume interactions between DPD particles of the corresponding types. As shown in Ref. [37], a standard choice of $a_{\alpha \alpha}=25$ [53] in insufficient to induce orientational ordering of NRs that are shorter than the copolymer domain width. Therefore we have taken $a_{\alpha \alpha}=50$ and continue to use it in the present study.

We describe the interaction of the NR $R$-particles with the polymer $A$ and $B$ particles by the selectivity parameter $\sigma=\left(a_{R B}-a_{R A}\right) /\left(a_{A B}-a_{R A}\right)$, which vanishes in the non-selective case $\left(a_{R B}=a_{R A}=50\right.$ in this study)

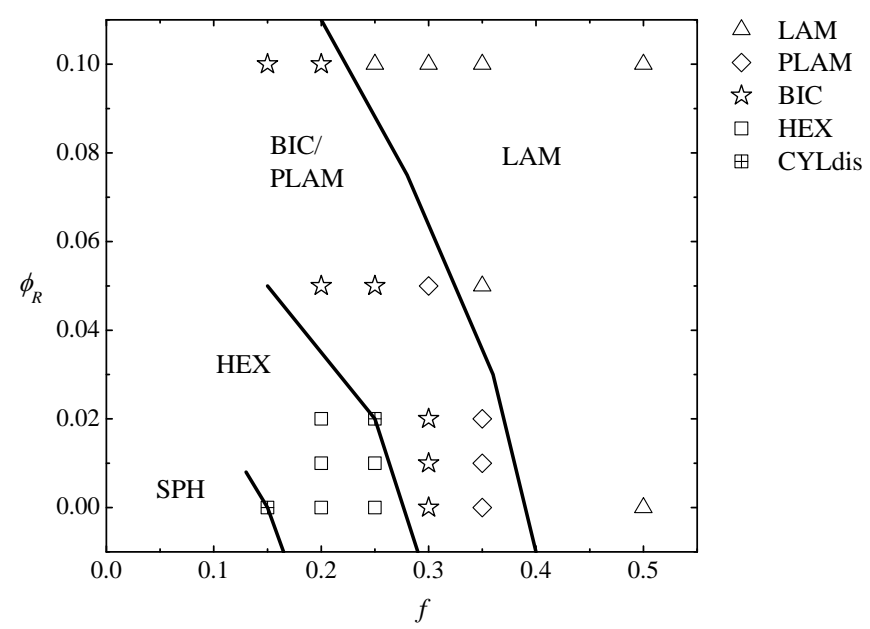

FIG. 6. Effect of the copolymer composition $f$ and the content of the particles $\phi_{R}$ on the morphology of the mixture with $N=20, N_{R}=5, \sigma=0.2$, and $a_{A B}=70$.
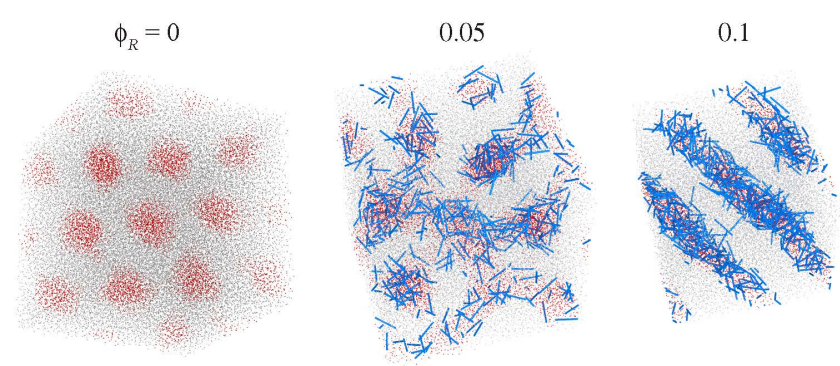

FIG. 7. Effect of the NR concentration $\phi_{R}$ on the microdomain structure at $f=0.25, \sigma=0.2, N=20, N_{R}=5$.

and increases to unity for the highest selectivity when $A$ and $R$ particles are identical and $a_{R B}=a_{A B}$. The overall volume fraction of NRs, $\phi_{R}$, takes small values from 0 to 0.1 to keep the copolymer matrix intact, while the average volume fractions of $A$ and $B$ units are equal to $\phi_{A}=\phi_{B}=\left(1-\phi_{R}\right) / 2$.

Microphase separation in the pure diblock copolymer $\mathrm{AB}$ and its composites with NRs is simulated by annealing the initially disordered structures upon a gradual, incremental (by 0.1 in the transition vicinity and by 1.0 in the strong segregation regime) increase in the repulsion parameter $a_{A B}$ between the particles $A$ and $B$. An order-disorder transition has been identified by a drop in the potential energy of the ordered system, appearance of a secondary peak in the static structure factor (the primary peak was shifted to $q \neq 0$ even in the disordered phase due to the composition fluctuations) and also visually from the structure snapshots (see Ref. [45] for details).

By resolving the equations describing the dynamics of the DPD liquid one can describe the stationary states of the composite in terms of the local volume frac- 
tions $\phi_{A}, \phi_{B}$ and $\phi_{R}$ of the monomer units $A$ and $B$ and the NRs, respectively. If the NRs are distributed within anisotropic microstructures formed by the diblock copolymer (namely, in lamellae and cylinders), one can also evaluate the degree of their orientational order in terms of the local nematic order parameter $S(\mathbf{r})$ identical to that defined by Eqs. (41) and (43):

$$
S(\mathbf{r})=\left\langle\frac{3}{2}[\mathbf{a}(\mathbf{r}) \cdot \mathbf{k}]^{2}-\frac{1}{2}\right\rangle,
$$

where $\mathbf{a}$ is a unit vector along the NR axis, $\mathbf{k}$ is the unit normal to the lamellar plane or to the cylindrical surface pointing in the direction of the NR center, and the angular brackets denote the averaging over a local subset of NRs. Zero value of the order parameter corresponds to the orientationally disordered NRs, whereas $S>0(S<$ 0 ) indicates their tendency to perpendicular (parallel) orientation with respect to the block boundary.

When a lamellar or cylindrical microstructure is formed, we are interested in the spatial profiles of all local variables along the normal to the layers or cylinders. However, in a fully-periodic simulation box it is impossible to predict the orientation of an emerging microstructure. In order to extract the information on the local distribution of the components from the simulation data, we apply the following procedure. We define the vectors $\mathbf{p}_{i}$ connecting the mass centres of $\mathrm{A}$ and B blocks in an $i$-th copolymer chain. For the lamellar case, such vectors are plotted for all copolymers in the system, then normalized to the unit length and translated to a common origin so that their ends form a cloud of $n \sim 10^{5}$ points non-uniformly distributed over the surface of a unit sphere. The gyration tensor of this cloud, $J_{\alpha \beta}=(1 / n) \sum_{i=1}^{n} p_{i \alpha} p_{i \beta}$ is diagonalized to find three eigenvalues and eigenvectors. The eigenvector corresponding to the largest eigenvalue is the vector $\mathbf{h}$, which is normal to the lamellar planes. In the case of cylinders, we consider a cloud of $\mathbf{q}_{i}=\mathbf{p}_{j} \times \mathbf{p}_{k}$ vectors and find the eigenvector corresponding to the largest eigenvalue of a tensor $J_{\alpha \beta}=(1 / n) \sum_{i=1}^{n} q_{i \alpha} q_{i \beta}$. This eigenvector is parallel to the axis of the cylinder and is perpendicular to the unit vector $\mathbf{h}$ which originates from the centre of a chosen NR. Below we select the $z$-axis in the direction of the vector $\mathbf{h}$. All local variables describing the composite structure are expressed as the functions of $z: \phi_{A}(z)$, $\phi_{B}(z), \phi_{A}(z), S(z)$, while the centre of a lamella or a cylinder is chosen as the origin $(z=0)$.

\section{B. Possible morphologies of the copolymer composite}

The variety of possible morphologies which have been found in the composite AB diblock copolymer $(N=20)$ doped with NRs $\left(N_{R}=5\right)$ is presented in Fig. 6 for various copolymer compositions $f$ and small $\left(\phi_{R}<0.1\right)$ NR volume fractions. NRs are taken to be weakly selective toward shorter copolymer blocks $(\phi=0.2)$, in which case the most pronounced effect is expected according to the preliminary studies. Another reason is that at $\phi=0.2$ the difference between alignment of NRs at the domain boundaries (parallel to the boundary) and in the domain bulk (perpendicular to the boundaries) is maximum for the symmetric copolymer [37]. In the absence of NRs $\left(\phi_{R}=0\right)$ a strongly-segregated AB diblock copolymer exhibits lamellae, perforated lamellae, a bicontinuous structure, cylindrical and spherical micellar structure depending on its composition [45]. One can see that the addition of NRs shifts the transitions between all ordered phases to more asymmetric compositions. As a result, spherical and cylindrical morphologies can be found only at a very small fraction of NRs $\left(\phi_{R}<0.05\right)$. The morphology of the copolymer with fixed composition $f$ may spontaneously change with the increasing NR concentration. This is shown in Fig. 7, where hexagonally packed cylinders in the pure diblock copolymer are replaced with the bicontinuous structure at $\phi_{R}=0.05$ and then with a lamellar structure at $\phi_{R}=0.1$ as predicted by the morphology diagram in Fig. 6 .

It should be noted that a rather wide domain of hexagonally perforated lamellar (a combination of 2D minor and 3D major component morphologies) and bicontinuous $(3 \mathrm{D} / 3 \mathrm{D})$ microstructures shown in Fig. 6 can represent non-equilibrium states. Although in pure diblock copolymers, self-consistent field theory [46] permits double gyroid and bicontinuous orthorhombic structures to be the only stable phases, yet the perforated lamellae phase has only slightly higher energy. Accordingly, the latter is often observed in laboratory experiments [47] and computer simulations, either by DPD [45] or Monte Carlo [48] methods, but is usually considered as a longliving metastable state. An example of such structure can be seen in the middle image in Fig. 7. However, it does not transform into a periodic gyroid phase in numerical simulations as this requires the DPD simulation cell size to be commensurate with the period of spontaneously forming microstructure [49].

\section{Positional and orientational ordering of nanorods in the composite}

The profiles of the local concentration and the orientational order parameter of the NRs in the lamellar phase are presented in Figs. 8 and 9 . It follows from Fig. 8 that highly selective NRs $(\sigma=1)$ are mainly located within the minor A domains and are preferentially aligned along the lamellae planes independently of their position in the domain. This is consistent with the predictions of the molecular theory presented above. Indeed, when a sufficiently long NR is inclined, it has a higher probability to cross the domain boundary, which is energetically unfavourable. In contrast, in the major B domains NRs are aligned perpendicular to the lamellae, which also confirms the predictions of the theory, but their concentration is very low. With a decrease in their 

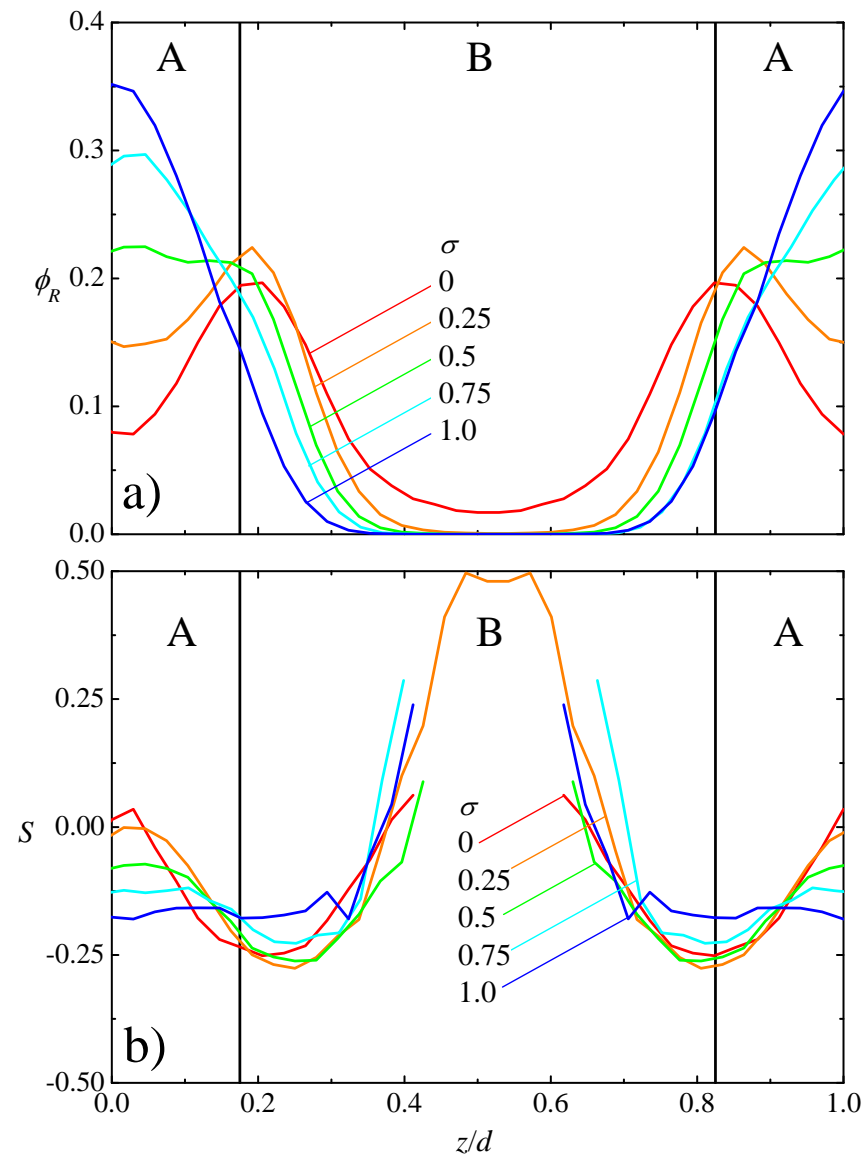

FIG. 8. Local fraction of the NRs $\phi_{R}(z)$ (a) and their orientational order parameter $S(z)$ (b) in the lamellar phase for different values of the NR interaction selectivity $\sigma$ (specified) for $f=0.35, \phi_{R}=0.1$, and $N_{R}=5$.

interaction selectivity, NRs accumulate near the domain boundaries which results in an increase of their orientational ordering. At the same time, in the bulk of the domains both the volume fraction and nematic order parameter are close to zero. One notes that the variation in the copolymer composition strongly affects the NR concentration profiles (see Fig. 9). In asymmetric copolymers, NRs are almost uniformly distributed within the minor phase while in the symmetric case they are mainly located at the domain boundaries. Penetration of the NRs effectively increases the volume fraction of the minor phase and makes the system more symmetric. The concentration and order parameter profiles of the NRs in the hexagonal phase are presented in Fig. 10. One can readily see that although the orientational order parameter is relatively low, it still possesses opposite signs in the domains A and B. Moreover, the concentration of NRs in domain $\mathrm{A}$ is comparable with that in domain $\mathrm{B}$ although in the lamellae phase the NRs of the same selectivity are predominantly located in domains A. One notes also that a small decrease of the domain $\mathrm{A}$ size results in a dramatic decrease of NR concentration in that domain
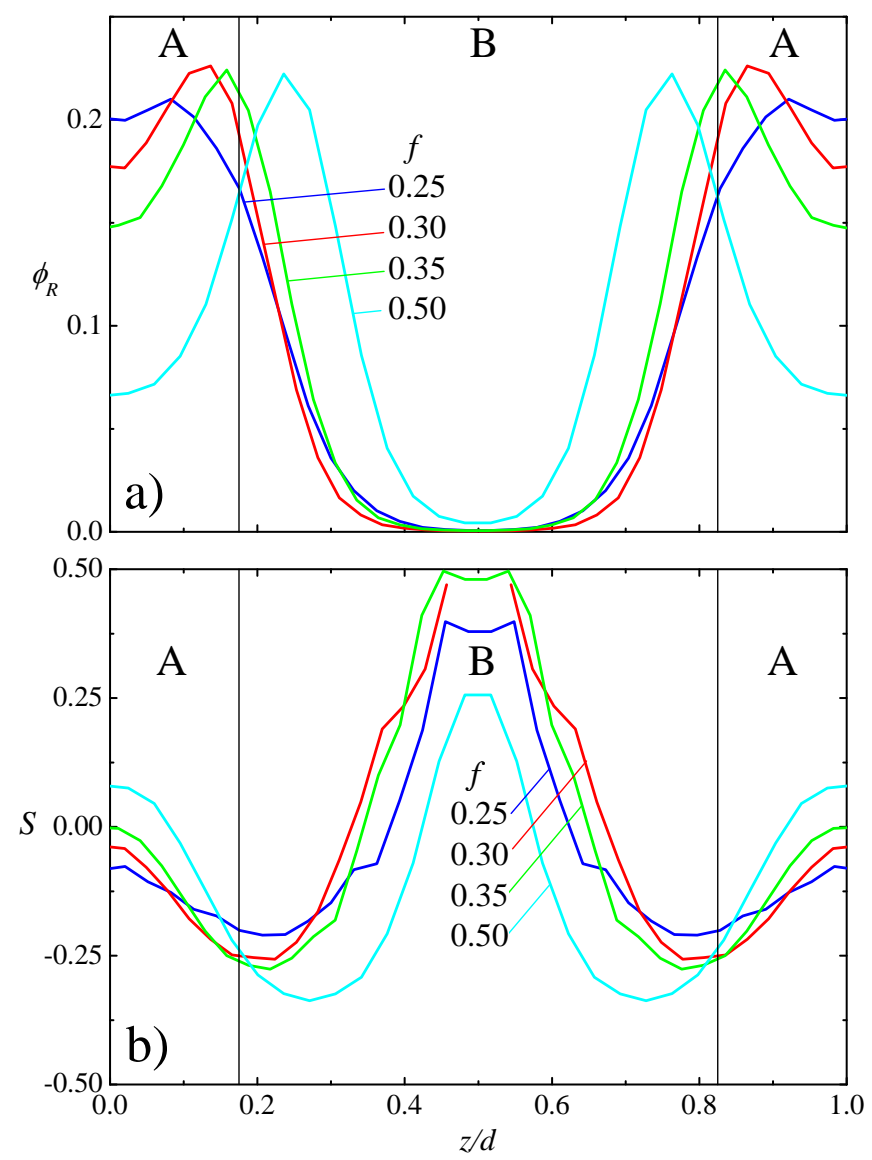

FIG. 9. Local fraction of the NRs $\phi_{R}(z)$ (a) and their orientational order parameter $S(z)$ (b) in the lamellar phase for different values of the copolymer composition $f$ (specified) for $\sigma=0.25, \phi_{R}=0.1$, and $N_{R}=5$.

despite the strong selectivity of the NRs. This can be explained by a significant decrease of the orientational entropy of long NRs located in a narrow domain which may overcome the decrease of the total interaction energy. The oscillations in the curves in Fig. 10 are related to the fact that the averaging has been undertaken over a single cylinder as different cylinders are not perfectly parallel in the simulations.

\section{DISCUSSION}

In this paper we have studied the properties of diblock copolymers doped with anisotropic NPs using a molecular mean-field theory and DPD computer simulations. The theory is based on the Landau expansion of the total free energy of the polymer nanocomposite in terms of the positional order parameter which describes the spatial distribution of monomers in the lamellae and the hexagonal phase. The coefficients of this expansion have been calculated using then mean-field theory taking into account the anisotropic interaction between NPs and the 

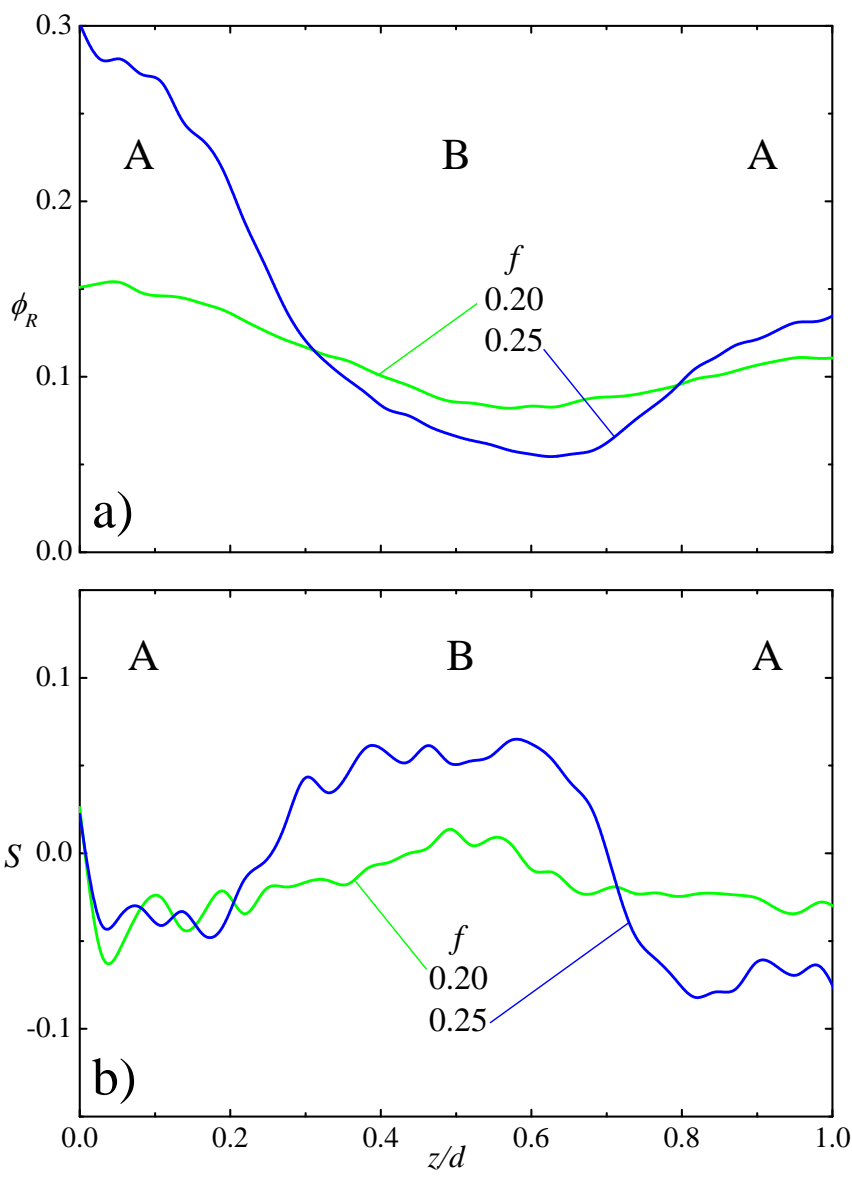

FIG. 10. Local fraction of the NRs $\phi_{R}(z)$ (a) and their orientational order parameter $S(z)$ (b) in the hexagonal cylindrical phase for different values of the copolymer composition $f$ (specified) for $\sigma=0.4, \phi_{R}=0.1$, and $N_{R}=5$.

monomers of both types. The anisotropic NP is modelled by a rigid system of two spheres separated by a certain distance, which interact isotropically with the monomers of the types A and B and the effective interaction potential between such a NP and the monomers is anisotropic, i.e., it depends on the angle between the intermolecular vector and the long NP axis. One notes that such an approach is generally valid only if the order parameter is relatively low and hence the theory can mainly be applied in the case of weak segregation. In contrast, the computer simulations are more effective in the case of strong segregation when the separation between different domains is more pronounced.

The effect of anisotropic NPs on the block copolymer composite is twofold. Firstly, the NPs are orientationally ordered (see Figs. 4, 5, 8, 9, and 10) in the boundary region between the blocks both in the lamellae and in the hexagonal phases and the corresponding orientational order parameter possesses opposite signs in adjacent blocks. This can be explicitly explained in the strongly segregated system, assuming, for instance, that the interaction of NPs with the monomers of the type $\mathrm{A}$ is stronger than the interaction with the monomers of the type B. Then, if the centre of a NP is located in the block A close to the boundary with the block B, the NP has a tendency to align parallel to the boundary in order to maximise its interaction with monomers $\mathrm{A}$ and to avoid contact with monomers B. In contrast, if such a $\mathrm{NP}$ is located in the block B close to its boundary, the total interaction energy is minimised when the NP is perpendicular to the boundary because in this case one of the interaction spheres is located in the block A which reduces the total interaction energy. In the case of weak segregation, the NPs are aligned by the gradients of the monomer concentration.

These qualitative results are supported by our computer simulations which employ the method of DPD and use the model of a NP composed of several interaction sites. One notes, however, that the theoretical values of the nematic order parameter appear to be rather low while the computer simulations predict values of about 0.5-0.6 which are comparable with those typical for nematic liquid crystals. This is partially explained by the effects of strong segregation. Large values of the nematic order parameter have also been obtained theoretically in our previous paper in the limit of infinitely strong segregation. Computer simulations also indicate that in the case of highly selective NPs they are aligned along the boundary independently of their location within the domain. This is related to the large length of NPs, used in the simulations, which is comparable to the domain size. In this case, the NP feels the boundary even when its centre is located in the bulk of a domain. The variation in the copolymer composition strongly affects the NP concentration profiles. In strongly asymmetric copolymers, the distribution of NPs within the minor phase is nearly homogeneous while in symmetric copolymers the NPs are mainly located at the domain boundaries.

In summary, both the molecular theory and computer simulations predict that anisotropic NPs are orientationally ordered in the lamellae as well as in the hexagonal phase and the corresponding orientational order parameter possess opposite signs in adjacent domains. As discussed in the Introduction, the two types of NP alignment have also been observed experimentally. At the same time, highly selective NPs are predominantly located in one of the domains and hence the majority of NPs in the composite are aligned along the same direction. This results in a sufficiently large dielectric and optical anisotropy of the nanocomposite if the NPs are polar and are characterised by a strong polarizability anisotropy. In principle, the regular microphase separation induces certain anisotropy of the macroscopic properties already in a pure block copolymer host. For example, it gives rise to the anisotropy of the chain statistics described by a specific tensor order parameter which can be also incorporated into the free energy expansion (7) following the guidelines of Ref. 55. However, the resulting anisotropy of the macroscopic properties is rather 
weak, as in the bulk of any domain the medium retains the local isotropy. In this context, adding even a small concentration of anisotropic NPs may result in a significant increase of the macroscopic dielectric anisotropy of the system due to the spontaneous orientational ordering of NPs. This effect opens the possibility to align such polymer nanocomposites by external fields which is very important for applications.

Secondly, NPs affect the stability ranges of the hexagonal, lamellae and disordered phases of block copolymers. As shown in Section III, the interaction between NPs and monomers renormalizes the quadratic term in the free energy expansion which results in the increase of the effective Flory discrimination parameter $\chi$. Thus the NPs generally stabilise the segregated phases. Parameters of the NPs also contribute to the higher order expansion coefficients and hence the effect of NPs can be rather complicated. In the case of weak segregation, NPs generally stabilise the hexagonal phase although at intermediate values of the polymer composition $f$ the range of the hexagonal phase expands only for sufficiently large selectivity and NP concentration. Computer simulations indicate that strongly-segregated diblock copolymers of different composition exhibit lamellae, perforated lamellae, bicontinuous, cylindrical and spherical micellar structures [45]. Addition of NPs shifts all phase transitions in the direction of lamellae or perforated lamellae phase. Moreover, the nanocomposite may undergo a transition between different phases with the increas- ing concentration of NPS $\phi_{R}$. For example, the composite diblock copolymer $(N=20)$ undergoes a transition from the hexagonal phase into the bicontinuous phase at $\phi_{R}=0.05$ and then into the lamellae phase at $\phi_{R}=0.1$ (see Fig. 7).

In general, block copolymer composites with anisotropic NPs can be considered as unconventional liquid crystal systems because they are characterised by the spontaneous orientational order of NPs. On the other hand, in contrast to nematic liquid crystals, this order is not determined by the anisotropic interaction between NPs themselves but is induced by the anisotropy of the block copolymer matrix (i.e. by the boundaries between the blocks). At higher concentration, the interaction between anisotropic NPs may become significant and then one may expect the emergence of the true liquid crystal order and the appearance of new macroscopic structures.

\section{ACKNOWLEDGMENTS}

M.A.O. and Y.V.K. are grateful to the Russian Science Foundation (project 16-13-10280), M.V.G. acknowledges the support from the Federal Agency of Scientific Organizations (Agreement No. 007-GZ/Ch3363/26). DPD simulations are carried out on the equipment of the shared research facilities of HPC computing resources at Lomonosov Moscow State University.
[1] Liquid Crystals with Nano and Microparticles, edited by J.P.F. Lagerwall and G. Scalia (World Scientific 2017).

[2] H. Qi, B. Kinkead, and T. Hegmann, Proc. SPIE 6911 , 691106 (2008).

[3] H. Qi and T. Hegmann, J. Mater. Chem. 16, 4197 (2006).

[4] Y. Shiraishi, N. Toshima, K. Maeda, H. Yoshikawa, J. $\mathrm{Xu}$, and S. Kobayashi Appl. Phys. Lett. 81, 2845 (2002).

[5] S.Kobayashi and N.Toshima, Information Display 23, 26 (2007).

[6] H. Yoshida, K. Kawamoto, H. Kubo, T. Tsuda, A. Fujii, S. Kuwabata, and M. Ozaki, Adv. Mater. 22, 622 (2010).

[7] Yu. Reznikov, O. Buchnev, O. Tereshchenko, V. Reshetnyak, A. Glushchenko, and J.West, Appl. Phys. Lett. 82, 1917 (2003).

[8] F. Li, O. Buchnev, C.I. Cheon, A. Glushchenko, V. Reshetnyak, Yu. Reznikov, T.J. Sluckin, and J.L. West, Phys. Rev. Lett. 97, 147801 (2006); Phys. Rev. Lett. 99, 219901(E) (2007).

[9] E. Ouskova, O. Bunchev, V. Reshetnyak, and Yu. Reznikov, Liq. Cryst. 30, 1235 (2003).

[10] S. Kaur, S. P. Singh, A. M. Biradar, A. Choudhary, and K. Sreeniva, Appl. Phys. Lett. 91, 023120 (2007).

[11] A. Kumar, J. Prakash, D. S. Mehta, A. M. Biradar, and W. Haase, Appl. Phys. Lett. 95, 023117 (2009).

[12] G.A. Shandryuk, E.V. Matukhina, R.B. Vasilev, A. Rebrov, G.N. Bondarenko, A.S. Merekalov, A.M Gas'kov, R.V. Tal'roze, Macromolecules 41, 2178 (2008).
[13] R.V. Talroze, G.A. Shandryuk, A.S. Merekalov, A.M. Shatalova, and O.A. Otmakhova, Polymer Science A 51, 1194 (2009).

[14] E.B. Barmatov, D.A. Pebalk, M.V. Barmatova, Liq. Cryst. 33, 1059 (2006).

[15] P. Kopcansky, N. Tomasovicova, M. Koneracka, M. Timko, Z. Mitroova, V. Zavisova, N. Eber, K. FodorCsorba, T. Toth-Katona, A. Vajda, J. Jadzyn, E. Beaugnon, X. Chaud, Acta Phys. Polonica A 118, 988 (2010).

[16] G. Sinha, C. Glorieux and J. Thoen Phys. Rev. E 69, 031707 (2004).

[17] T. Bellini, M. Buscaglia, C. Chiccoli, F. Mantegazza, P. Pasini, and C. Zannoni Phys. Rev. Lett. 85, 1008 (2000)

[18] H. Duran, B. Gazdecki, A. Yamashita and T. Kyu, Liq. Cryst. 32, 815 (2005).

[19] P. Kopcansky, N. Tomasovicova, M. Koneracka, M. Timko, V. Zavisova, A. Dzarova, J. Jadzyn, E. Beaugnon, and X. Chaud, Int. J. Thermophys. 32, 807 (2011).

[20] M.V. Gorkunov and M.A. Osipov, Soft Matter 7, 4348 (2011).

[21] L.M. Lopatina and J.V. Selinger, Phys. Rev. E 84, 041703 (2011).

[22] L.M. Lopatina and J.V. Selinger, Phys. Rev. Lett. 102, 197802 (2009).

[23] M.A. Osipov and M.V. Gorkunov, ChemPhysChem. 15, 1496 (2014). 
[24] M.A. Osipov and M.V. Gorkunov, Phys. Rev. E 92, 032501 (2015).

[25] M. Alexandre, P. Dubois, Mater. Sci. Eng. Rev. 28, 1 (2000).

[26] A.C. Balazs, Curr. Opin. Colloid Interface Sci. 4, 443 (2000).

[27] E.P. Giannelis, Appl. Organomet. Chem. 12, 675 (1998).

[28] P.P. Soo, B.Y. Huang, Y.I. Jang, Y.M. Chiang, D.R. Sadoway, A.M. Mayes, J. Electrochem. Soc. 146, 32 (1999).

[29] A. C. Balazs, T. Emrick, T. P. Russell, Science 3141107 (2006).

[30] R. Krishnamoorti, R. A. Vaia, J. Polym. Sci., Part B: Polym. Phys. 45, 3252 (2007).

[31] A. J. Crosby and J. Y. Lee, Polym. Rev. 47, 217 (2007).

[32] R.D. Deshmukh, Y. Liu, R.J. Composto, Nano Lett. 7, 3662 (2007).

[33] K. Thorkelsson, A.J. Mastroianni, P. Ercius, T. Xu, Nano Lett. 12, 498 (2012)

[34] E. Ploshnik, A. Salant, U. Banin, R. Shenhar, Adv. Mater. 22, 2774 (2010).

[35] E. Ploshnik, A. Salant, U. Banin, R. Shenhar, Phys. Chem. Chem. Phys. 12, 11885 (2010).

[36] M.A. Osipov and M.V. Gorkunov, Eur. Phys. J. E 39, 126 (2016).

[37] A.V. Berezkin, Y.V. Kudryavtsev, M.V. Gorkunov, and M.A. Osipov, J. Chem. Phys. 146, 144902 (2017).

[38] M.A. Osipov, A.S. Ushakova, and M.V. Gorkunov, Liq. Cryst. 44, 1861 (2017).

[39] G. H. Fredrickson, V. Ganesan and F. Drolet, Macromolecules 35, 16 (2002); M. W. Matsen, J. Phys.: Condens. Matter 14, R21 (2002).
[40] K. Hur, R.G. Hennig, F.A. Escobedo, and U. Wiesner, J. Chem. Phys. 133, 194108 (2010).

[41] B. Rasin, H. Chao, G. Jiang, D. Wang, R. A. Riggleman and R. J. Composto, Soft Matter 12, 2177 (2016), Q.Y. Tang and Y.-Q. Ma, J. Phys. Chem. B 113, 10117 (2009).

[42] L. Leibler, Macromolecules 13, 1602 (1980).

[43] T. Ohta, K. Kawasaki, Macromolecules 19, 2621 (1986); ibid. 23, 2413 (1990).

[44] T. Uneyama, M. Doi, Macromolecules 38, 196 (2005).

[45] A. A. Gavrilov, Y. V. Kudryavtsev and A. V. Chertovich, J. Chem. Phys. 139, 224901 (2013).

[46] M. W. Matsen, M. Schick, Phys. Rev. Lett. 72, 2660 (1994).

[47] V. Abetz, P. Simon, Adv. Polym. Sci. 189, 125 (2005).

[48] T.M. Beardsley, M.W. Matsen, Eur. Phys. J. E, 32, 255 (2010).

[49] F. J. Martinez-Veracoechea, F. A. Escobedo, J. Chem. Phys. 125, 104907 (2006).

[50] L.-T. Yan and X.-M. Xie, Prog. Polym. Sci. 38, 369 (2013).

[51] http://lammps.sandia.gov/

[52] G. Besold, I. Vattulainen, M. Karttunen, and J. M. Polson, Phys. Rev. E 62, R7611 (2000).

[53] R. D. Groot and P. B. Warren, J. Chem. Phys. 107, 4423 (1997).

[54] T. F. Miller, M. Eleftheriou, P. Pattnaik, A. Ndirango, D. Newns and G. J. Martyna, J. Chem. Phys. 116, 8649 (2002).

[55] S. Yabunaka, T. Ohta, Soft Matter 9, 7479 (2013). 